\title{
CONVERGENCE METHOD, PROPERTIES AND COMPUTATIONAL COMPLEXITY FOR LYAPUNOV GAMES
}

\author{
Julio B. CLEMPNER *, AleXANDer S. POZNYAK** \\ * Center for Computing Research, National Polytechnic Institute \\ Av. Juan de Dios Batiz s/n, Edificio CIC, Col. Nueva Industrial Valleys, 07738 Mexico City, Mexico \\ e-mail: julio@clempner.name \\ ** Department of Automatic Control, Center for Research and Advanced Studies \\ Av. IPN 2508, Col. San Pedro Zacatenco, 07360 Mexico City, Mexico \\ e-mail: apoznyak@ctrl.cinvestav.mx
}

\begin{abstract}
We introduce the concept of a Lyapunov game as a subclass of strictly dominated games and potential games. The advantage of this approach is that every ergodic system (repeated game) can be represented by a Lyapunov-like function. A direct acyclic graph is associated with a game. The graph structure represents the dependencies existing between the strategy profiles. By definition, a Lyapunov-like function monotonically decreases and converges to a single Lyapunov equilibrium point identified by the sink of the game graph. It is important to note that in previous works this convergence has not been guaranteed even if the Nash equilibrium point exists. The best reply dynamics result in a natural implementation of the behavior of a Lyapunov-like function. Therefore, a Lyapunov game has also the benefit that it is common knowledge of the players that only best replies are chosen. By the natural evolution of a Lyapunov-like function, no matter what, a strategy played once is not played again. As a construction example, we show that, for repeated games with bounded nonnegative cost functions within the class of differentiable vector functions whose derivatives satisfy the Lipschitz condition, a complex vector-function can be built, where each component is a function of the corresponding cost value and satisfies the condition of the Lyapunov-like function. The resulting vector Lyapunov-like function is a monotonic function which can only decrease over time. Then, a repeated game can be represented by a one-shot game. The functionality of the suggested method is successfully demonstrated by a simulated experiment.
\end{abstract}

Keywords: Lyapunov game, Lyapunov equilibrium point, best reply, repeated games, forward decision process.

\section{Introduction}

There are several disadvantages in the use of Nash equilibria (Goemans et al., 2005). The use of pure strategies implies that pure Nash equilibria may not exist in a game while, on the other hand, the use of mixed strategies to find the equilibria do not particularly correspond to acknowledged facts and sometimes represent an artificial solution of the game. Another constraint is related to the prior knowledge of the equilibrium point. Bellman's equation is expressed as a sum over the state of a trajectory needs to be solved backwards in time from the target point. One of the most interested drawbacks of Nash equilibria is related to the convergence and stability of equilibrium points. Nash equilibria are considered a solution of a game if the system arrives at such stable points. But, in many games convergence to Nash equilibria is not as- sured. In this sense, the most natural approach for finding a Nash equilibrium of a given game is executing the best reply dynamics. Even in repeated games where alternative mechanisms for equilibrium play are proposed the convergence is not guaranteed.

Another disadvantage is the proof that finding a Nash equilibrium is an intractable problem (Chen and Deng, 2006; Daskalakis et al., 2006a) which motivates efforts aimed at presenting an approximate Nash equilibrium as an alternative solution (Lipton et al., 2003; Daskalakis et al., 2006b; Kontogiannis et al., 2006), although negative results about it arise as well (Chen et al., 2006; Daskalakis et al., 2006b). For these reasons we propose an alternative solution concept to analyze and improve such drawbacks. In this paper we introduce the concept of a Lyapunov game (Clempner, 2006) as a subclass of strictly dominated games and potential games, analyzing its convergence and 
complexity properties. We propose an alternative solution concept focusing on a class of games for which Lyapunov theory is naturally applied and the convergence is guaranteed by a Lyapunov-like function.

In particular, we focus on strictly dominated games, also called the iterated dominance equilibrium (Bernheim, 1984; Moulin, 1984; Pearce, 1984), in which a strategy profile can be found by deleting a dominated strategy from the strategy set of one of the players, recalculating to find which remaining strategies are dominated, deleting one of them, and continuing the process until only one strategy remains for each player. The best reply dynamics result in a natural implementation of the behavior of a Lyapunovlike function. The dynamics begin choosing an arbitrary strategy profile of the players (Nash, 1951; 1996; 2002; Myerson, 1978; Selten, 1975). Then, in each step of the process some player exchanges his/her strategy to be the best reply to the current strategies of the other players. A Lyapunov-like function monotonically decreases and it results in the elimination of a strictly dominated strategy from the strategy space. As a consequence, the problem complexity is reduced. In the next step, the strategies that survived the first elimination round and are not best replies to some strategy profile are eliminated, and so forth. This process finishes when the Lyapunov-like function converges to a Lyapunov equilibrium point. It is important to note that by the natural evolution of a Lyapunov-like function, if a strategy was played once it is not played again.

The dynamics of a game are represented by a directed graph $G$, where an edge $\left(s_{i}, s_{j}\right)$ means that $s_{i}$ has a higher payoff than $s_{j}$, i.e., $\mathbb{V}\left(s_{j}\right)<\mathbb{V}\left(s_{i}\right)$ (except at the sink, at an equilibrium point, where $\mathbb{V}\left(s_{i}\right)=\mathbb{V}\left(s_{j}\right)$ ). In a more restrictive version, $s_{j}$ represents the best response to $s_{i}$. The evolution of the game will be represented by a path in $G$. Such a path may converge to a pure equilibrium. The pure equilibrium is the sink of the graph $G$.

In Lyapunov games the natural existence of the equilibrium point is assured by definition. In this sense, fixedpoint conditions for games are given by the definition of the Lyapunov-like function, in contrast to the fact that a Nash equilibrium must satisfy Kakutani's fixed-point theorem (Kakutani, 1941). We claim that a Lyapunov game has a single Lyapunov equilibrium point (by definition).

It is important to note that convergence is also guaranteed. A kind of discrete vector can be imagined over the game graph. Each optimal action applied yields a reduction in the optimal cost-to-target value, until the equilibrium point is reached by the definition of the Lyapunovlike function. It is important to note that a Lyapunov-like function is constructed to respect the constraints imposed by the system.

When a (repeated) game and its strategies are played over and over, a learning mechanism is implemented to justify an equilibrium play (Poznyak et al., 2000). Un- fortunately, in "classical games", the convergence is not guarantees even if a Nash equilibrium point exists. On the other hand, in one-shot games it is difficult for the players to identify the correct expectations about the strategy choices of their opponents. In our case, the problem is more difficult to justify because repeated games are transformed to one-shot games replacing the learning mechanism by a Lyapunov-like function. However, a Lyapunovlike function definitely converges to a Lyapunov equilibrium point (Clempner, 2006).

An important advantage of Lyapunov games is that every ergodic system can be represented by a Lyapunovlike function. The function replaces the recursive mechanism with the elements of the ergodic system that model how players are likely to behave in one-shot games. As a construction example, we first propose a non-converging state-value function that fluctuates (increases and decreases) between states of the game. Then, we show that for repeated games with bounded nonnegative cost functions within the class of differentiable vector-functions whose derivatives satisfy the Lipschitz condition, a complex vector-function can be built where each component is a function of the corresponding cost-value and satisfies the condition of the Lyapunov-like function. The resulting cost-value function is a monotonic function which can only decrease (or remain the same) over time.

The optimal discrete problem is computationally tractable. The cost-to-target values are calculated using a Lyapunov-like function. The Lyapunov-like function is used as a forward trajectory-tracking function. Every time a discrete vector field of possible actions is calculated over the game. Each optimal action applied decreases the optimal value, ensuring that the optimal course of action is followed establishing a preference relation.

1.1. Organization of the paper. The remainder of this paper is organized as follows. The next section presents the necessary mathematical background and terminology needed to understand the paper. Section 3 introduces the Lyapunov game definition and derives conditions for the uniqueness of the equilibrium point. Section 4 contains the main technical results concerning to the fact that every ergodic system (repeated game) can be represented by a Lyapunov-like function, presenting the corresponding simulated experiments, and the complexity for reaching a Lyapunov (Nash) equilibrium point is also calculated. Finally, in Section 5 some concluding remarks and future work projects are outlined.

\section{Preliminaries}

2.1. Game description. In general, a non-cooperative game is the triple $G=\left\langle\mathcal{N},\left(S_{\iota}\right)_{\iota \in \mathcal{N}},\left(\leq_{\iota}\right)_{\iota \in \mathcal{N}}\right\rangle$, where

- $\mathcal{N}=\{1,2, \ldots, n\}$ is a finite set of players, 
- $S_{\iota}$ is a finite set of "pure" strategies (henceforth called actions) of each player $\iota \in \mathcal{N}$,

- $\left(\leq_{\iota}\right)$ is a binary relationship over $S:=\prod_{\iota \in \mathcal{N}} S_{\iota}$ reflecting the preferences of the player $t$ over the outcomes.

It is assumed that the relation $\left(\leq_{\iota}\right)$ establishes a poset on $S$, i.e., given $r, s, t \in S$, we expect the preference relation $\left(\leq_{\iota}\right)$ to be fulfilled, and the following axioms hold: reflexivity $\left(r \leq_{\iota} r\right)$, antisymmetry $\left(r \leq_{\iota} s\right.$ and $s \leq_{\iota} r$ implies that $r=s)$, transitivity $\left(r \leq_{\iota} s\right.$ and $s \leq_{\iota} t$ implies that $r \leq_{\iota} t$ ).

Although the preference relation is the basic primitive of any decision problem (and generally observable), it is much easier to work with a consistent cost function,

$$
\mathbb{U}_{\iota}: S \rightarrow \mathbb{R}_{+},
$$

because we only have to use $n$ real numbers

$$
\mathbb{U}=\left\{\mathbb{U}_{1}, \ldots, \mathbb{U}_{n}\right\} .
$$

Definition 1. The cost function $\mathbb{U}_{\iota}(1)$ is said to be consistent with the preference relationship $\left(\leq_{\iota}\right)$ of a decision problem $(S, \leq)$ if and only if for any $s, t \in S$

$$
\mathbb{U}_{\iota}(s) \leq \mathbb{U}_{\iota}(t)
$$

which shortly is denoted as

$$
s \leq_{\mathbb{U}_{\iota}} t
$$

Denote by $G=\left\langle\mathcal{N},\left(S_{\iota}\right)_{\iota \in \mathcal{N}},\left(\mathbb{U}_{\iota}\right)_{\iota \in \mathcal{N}}\right\rangle$ the corresponding game.

For notational convenience we write $S=\prod_{\iota \in \mathcal{N}} S_{\iota}$ understanding the pure strategies profile, and $S_{-\iota}=$ $\prod_{j \in \mathcal{N} \mid\{\iota\}} S_{j}$, the pure strategies profile of all the players except the player $\iota$. For an action tuple $s=$ $\left(s_{1}, \ldots, s_{n}\right) \in S$ we denote the complement action as $s_{-\iota}=\left(s_{1}, \ldots, s_{\iota-1}, s_{\iota+1}, \ldots, s_{n}\right)$ and, with an abuse of notation, $s=\left(s_{\iota}, s_{-\iota}\right)$.

2.2. Association with a direct acyclic graph. Let us associate to any game $G=\left\langle\mathcal{N},\left(S_{\iota}\right)_{\iota \in \mathcal{N}},\left(\mathbb{U}_{\iota}\right)_{\iota \in \mathcal{N}}\right\rangle$ a $d i$ rect acyclic graph (Topkis, 1979). At this point let us introduce some notation on partial order.

For any $s \in S$, let

- successors of $s$ :

$$
\begin{aligned}
& t \in \operatorname{suc}(s) \text { iff } s \neq t, s \leq \mathbb{U}_{\iota} t \text { and } \\
& \forall q:\left(s \leq_{\mathbb{U}_{\iota}} q \leq_{\mathbb{U}_{\iota}} t\right) \Longrightarrow(q=s) \vee(q=t) ;
\end{aligned}
$$

- predecessors of $s$ :

$$
\begin{aligned}
& t \in \operatorname{pre}(s) \text { iff } t \neq s, t \leq \mathbb{U}_{\iota} s \text { and } \\
& \forall q:\left(t \leq_{\mathbb{U}_{\iota}} q \leq_{\mathbb{U}_{\iota}} s\right) \Longrightarrow(q=t) \vee(q=s) .
\end{aligned}
$$

Let $G_{\mathbb{U}}$ be the graph whose set of nodes is $S$. For each pair $(s, t) \in S^{2}:(s, t)$ is an edge iff $t \in \operatorname{suc}(s)$ or, equivalently, $s \in$ pre $(t)$.

Definition 2. We say that $\mathbb{U}$ is consistent with the preference relation $\left(\leq_{\mathbb{U}}\right)$ if $G_{\mathbb{U}}$ has no cycles, namely, $G_{\mathbb{U}}$ is a direct acyclic graph.

From now on, we will consider only consistent cost functions. Obviously,

$$
\forall s, t \in S:\left(s<_{\mathbb{U}_{\iota}} t\right) \vee\left(s \equiv_{\mathbb{U}_{\iota}} t\right) \vee\left(t<\mathbb{U}_{\iota} s\right)
$$

Thus, $\mathbb{U}_{\iota}$ induces a hierarchical structure on $S$.

The maximal elements are those with no predecessors, i.e., nodes with a null inner degree in $G_{\mathbb{U}}$. The minimal elements are those with no successors, i.e., nodes with a null outer degree in $G_{\mathbb{U}}$.

Define the upper distance $d^{+}$among actions $(s, t) \in$ $S^{2}$ as follows:

$$
\begin{aligned}
& d^{+}(s, t)=1 \Longleftrightarrow t \in \operatorname{suc}(s), \\
& d^{+}(s, t)=1+r \Longleftrightarrow \\
& \exists q: d^{+}(s, q)=r \& d^{+}(q, t)=1 .
\end{aligned}
$$

Similarly, the lower distance $d^{-}$among actions $(s, t) \in S^{2}$ satisfies

$$
\begin{aligned}
& d^{-}(s, t)=1 \Longleftrightarrow t \in \operatorname{pre}(s), \\
& d^{-}(s, t)=1+r \Longleftrightarrow \\
& \exists q: d^{-}(s, q)=r \& d^{-}(q, t)=1 .
\end{aligned}
$$

Thus

$$
d^{+}(s, t)=d^{-}(t, s)
$$

The upper height of a node $s$ is

$$
h^{+}(s)=\max _{t: t \text { is maximal }} d^{+}(s, t) .
$$

The lower height of a node $s$ is

$$
h^{-}(s)=\max _{t: t \text { is minimal }} d^{-}(s, t) .
$$

\subsection{Individual and common hierarchies.}

Definition 3. Let $S \neq \emptyset, \mathbb{U}_{\iota}: S_{\iota} \rightarrow \mathbb{R}_{+}$and $\mathbb{U}_{\kappa}: S_{\kappa} \rightarrow$ $\mathbb{R}_{+}$be two real vector-functions. We say that

(a) $\mathbb{U}_{\iota}$ is an $(\iota, \kappa)$-eq-order of $\mathbb{U}_{\kappa}$ if

$$
\begin{aligned}
\forall s_{1}, s_{2} \in S:\left(\mathbb{U}_{\iota}\left(s_{1}\right)\right. & \left.=\mathbb{U}_{\iota}\left(s_{2}\right)\right) \\
& \Longrightarrow\left(\mathbb{U}_{\kappa}\left(s_{1}\right)=\mathbb{U}_{\kappa}\left(s_{2}\right)\right) .
\end{aligned}
$$

(In this case, $\left(S / \equiv_{\mathbb{U}_{\iota}}\right)$ is a homeomorphic image of $\left(S / \equiv_{\mathbb{U}_{\kappa}}\right)$ since both are linearly ordered sets.) 
(b) $\mathbb{U}_{\iota}$ is an $(\iota, \kappa)$-ineq-order of $\mathbb{U}_{\kappa}$ if

$$
\begin{aligned}
\forall s_{1}, s_{2} \in S:\left(\mathbb{U}_{\iota}\left(s_{1}\right)\right. & \left.\leq \mathbb{U}_{\iota}\left(s_{2}\right)\right) \\
& \Longrightarrow\left(\mathbb{U}_{\kappa}\left(s_{1}\right) \leq \mathbb{U}_{\kappa}\left(s_{2}\right)\right),
\end{aligned}
$$

(In this case, the ordering $\leq_{\mathbb{U}}$, is included, as a set, in the ordering $\leq_{\mathbb{U}_{\kappa}}$.) Hence, $G_{\mathbb{U}_{\kappa}}$ is homomorphic image of $G_{\mathbb{U}_{\iota}}$, i.e., $G_{\mathbb{U}_{\kappa}}$ can be realized as a subgraph of $G_{\mathbb{U}_{L}}$

(c) $\mathbb{U}_{\iota}$ is an $(\iota, \kappa)$-tonal-order of $\mathbb{U}_{\kappa}$ if

$$
\begin{aligned}
& \forall s_{1}, s_{2} \in S: \operatorname{sgn}\left(\mathbb{U}_{\iota}\left(s_{1}\right)-\mathbb{U}_{\iota}\left(s_{2}\right)\right) \\
& =\operatorname{sgn}\left(\mathbb{U}_{\kappa}\left(s_{1}\right)-\mathbb{U}_{\kappa}\left(s_{2}\right)\right),
\end{aligned}
$$

where sgn $: \mathbb{R} \rightarrow \mathbb{R}$ is defined as

$$
\operatorname{sgn}\left(x_{t}\right):=\left\{\begin{array}{clc}
1 & \text { if } & x_{t}>0 \\
0, & \text { if } & x_{t}=0 \\
-1, & \text { if } & x_{t}<0
\end{array}\right.
$$

(In this case, $G_{\mathbb{U}_{\kappa}}$ is isomorphic to $G_{\mathbb{U}_{\iota}}$.)

Given two cost-functions $\mathbb{U}_{\iota}, \mathbb{U}_{\kappa}: S \rightarrow \mathbb{R}_{+}$it is interesting to decide whether there is a common hierarchy to the hierarchies in $S$ induced by individual cost functions $\mathbb{U}_{\iota}, \mathbb{U}_{\kappa}$ that, in fact, defines how the game considered is realized. We may proceed with two equivalent approaches.

\subsubsection{Common hierarchy construction based on the} "product" of individual orders. Let $\mathbb{R}^{N}$ be ordered with the product of the usual ordering in $\mathbb{R}$ (lexicographic), namely,

$$
\begin{aligned}
& \left(a_{1}, a_{2}, \ldots, a_{p}\right) \leq\left(b_{1}, b_{2}, \ldots, b_{p}\right) \\
& \Leftrightarrow(\exists m>0)(\forall i>m)\left(a_{i}=b_{i}\right) \wedge\left(a_{m}<_{m} b_{m}\right) .
\end{aligned}
$$

Then

$$
\begin{aligned}
\forall s, s^{\prime} & \left.\in S: s \leq \mathbb{U}_{1}, \mathbb{U}_{2}, \ldots, \mathbb{U}_{n}\right) \\
\quad & s^{\prime} \\
& \leq\left(\mathbb{U}_{1}(s), \mathbb{U}_{2}(s), \ldots, \mathbb{U}_{n}(s)\right) \\
& \leq\left(\mathbb{U}_{1}\left(s^{\prime}\right), \mathbb{U}_{2}\left(s^{\prime}\right), \ldots, \mathbb{U}_{n}\left(s^{\prime}\right)\right),
\end{aligned}
$$

and hence, by this ordering, we construct a graph $G_{\left(\mathbb{U}_{1}, \mathbb{U}_{2}, \ldots, \mathbb{U}_{n}\right)}$ on $S$.

2.3.2. Common hierarchy construction based on the "union" of individual orders. Let $G_{\mathbb{U}_{\iota}}$ and $G_{\mathbb{U}_{\kappa}}$ be graphs on $S$ obtained by functions $\mathbb{U}_{\iota}$ and $\mathbb{U}_{k}$, respectively. Let $G_{\mathbb{U}_{\iota} * \mathbb{U}_{\kappa}}$ be the union of $G_{\mathbb{U}_{\iota}}$ and $G_{\mathbb{U}_{\kappa}}$, that is,

$\left((s, t)\right.$ is an edge of $\left.G_{\mathbb{U}_{\iota} * \mathbb{U}_{\kappa}}\right) \Leftrightarrow$

$\left((s, t)\right.$ is an edge of $\left.G_{\mathbb{U}_{\iota}}\right) \vee\left((s, t)\right.$ is an edge of $\left.G_{\mathbb{U}_{\kappa}}\right)$.

$G_{\mathbb{U}_{\iota} * \mathbb{U}_{\kappa}}$ has no cycles provided that no $\left(G_{\mathbb{U}_{\iota}}\right.$ and $\left.G_{\mathbb{U}_{\kappa}}\right)$ has cycles. Nevertheless, this condition is not sufficient to obtain $G_{\mathbb{U}_{\iota} * \mathbb{U}_{\kappa}}$ free of cycles.

\section{Lyapunov games}

3.1. Vector Lyapunov-like functions. Let $G(\mathcal{V}, \mathcal{E})$ be referred to as a game graph such that the nodes $\mathcal{V}$ are elements of $S$ and the edges $\mathcal{E}$ are

$$
\mathcal{E}=\left\{\left(s, s^{\prime}\right): \exists \iota s^{\prime}=\left(s_{\iota}^{\prime}, s_{-\iota}\right) \wedge s^{\prime} \leq_{\mathbb{U}} s\right\} .
$$

A sink (see, e.g., Goemans et al., 2005; Mirrokni and Vetta, 2004; Fabrikant et al., 2004; Fabrikant and Papadimitriou, 2008), is a node with no outer degree (no outgoing edges) in the game graph $G$. The next definition will be in force throughout the paper.

Definition 4. Let $\mathbb{V}: S \rightarrow \mathbb{R}_{+}^{N}$ be a vector continuous map. Then, $\mathbb{V}$ is said to be a vector Lyapunov-like function (see, e.g., Lakshmikantham et al., 1991) associated with the given game $G(\mathcal{V}, \mathcal{E})$ iff it satisfies the following properties :

(1) there is a $s^{*}$, called below a Lyapunov equilibrium point such that $\mathbb{V}_{\iota}\left(s^{*}\right)=0$;

(2) $\mathbb{V}_{\iota}(s)>0$ for all $s \neq s^{*}$ and all $\iota \in \mathcal{N}$;

(3) $\mathbb{V}_{\iota}(s) \rightarrow \infty$ as $s \rightarrow \infty$ for all $\iota \in \mathcal{N}$;

(4) $\Delta \mathbb{V}_{\iota}=\mathbb{V}_{\iota}\left(s^{\prime}\right)-\mathbb{V}_{\iota}(s)<0$ for all $s^{\prime} \leq_{\mathbb{V}} s: s, s^{\prime} \neq$ $s^{*}$ and all $\iota \in \mathcal{N}$.

Now we are ready to formulate the first important proposition concerning the game $G(\mathcal{V}, \mathcal{E})$.

Definition 5. An equilibrium point $s^{*} \in \mathcal{V}$ with respect the game graph $G(\mathcal{V}, \mathcal{E})$ is a sink node.

Proposition 1. Let the set $S$ be finite and $\mathbb{V}$ be a vector Lyapunov-like function associated with the given game $G(\mathcal{V}, \mathcal{E})$. Then $\mathbb{V}$ are consistent with the preference relation $(\leq \mathbb{V})$

Proof. Let $\left(\equiv_{\mathbb{V}}\right)$ be the equivalence relation on $S$ induced by $\mathbb{V}$ :

$$
\forall s, t \in S: s \equiv_{\mathbb{V}} t \Longleftrightarrow \mathbb{V}(s)=\mathbb{V}(t) .
$$

Then the collection of equivalence classes

$$
\left\{S / \equiv_{\mathbb{V}}\right\}=\bigcup_{\iota \in \mathcal{N}} S / \equiv_{\mathbb{V}}=\{\pi(s) \mid s \in S\},
$$

where $\pi(s)$ is a partition on $S$, is a poset isomorphic to a subset of $\mathbb{R}$. Thus, $\left\{S / \equiv_{\mathbb{V}}\right\}$ is linearly ordered and, consequently, it is a lattice. The structure $\left\{S / \equiv_{\mathbb{V}}\right\}$ is indeed trivial: all elements in $S$ giving the same value under $\mathbb{V}$ are identified in this quotient set. On the other hand, for the relation $\left(\leq_{\mathbb{V}}\right)$ the following holds:

$$
\forall s, t \in S: s \leq_{\mathbb{V}} t \Longleftrightarrow \mathbb{V}(s) \leq \mathbb{V}(t) .
$$

This relation is reflexive and transitive, and it is antisymmetric since the Lyapunov-like function $\mathbb{V}$ is a oneto-one mapping. Thus, $\left(\leq_{\mathbb{V}}\right)$ is an ordering in $S$.

Corollary 1. Let $G_{\mathbb{V}}$ be the graph induced by the Lyapunov-like functions $\mathbb{V}$. Then $G_{\mathbb{V}}$ is free of cycles. 
3.2. Best reply strategies. Consider a game graph $G(\mathcal{V}, \mathcal{E})$

Definition 6. A strategy $\delta_{\iota} \in S_{\iota}$ is said to be the best reply to a given strategy-profile of the other players $s_{-\iota}=$ $\left(s_{1}, \ldots, s_{\iota-1}, s_{\iota+1}, \ldots, s_{n}\right)$ if for all $s_{\iota} \in S_{\iota}$ we have that $\mathbb{V}\left(\delta_{\iota}, s_{-\iota}\right) \leq \mathbb{V}\left(s_{\iota}, s_{-\iota}\right)$ (in the component-wise sense). For each player $\iota \in \mathcal{N}$ and each strategy-profile of the other players $s_{-\iota} \in S_{-\iota}$ denote the set of best replies by $b_{\iota}\left(s_{-\iota}\right)$, i.e., the set of actions that player $\iota$ cannot improve upon:

$$
\begin{aligned}
& b_{\iota}\left(s_{-\iota}\right) \\
& \quad:=\left\{\delta_{\iota} \in S_{\iota} \mid \forall s_{\iota} \in S_{\iota}: \mathbb{V}_{\iota}\left(\delta_{\iota}, s_{-\iota}\right) \leq \mathbb{V}_{\iota}\left(s_{\iota}, s_{-\iota}\right)\right\} .
\end{aligned}
$$

A strategy $\delta_{\iota} \in S_{\iota}$ is called a never best reply if

$$
\min _{\delta_{\iota} \in b_{\iota}\left(s_{-\iota}\right)} \mathbb{V}\left(\delta_{\iota}, s_{-\iota}\right)<\mathbb{V}\left(s_{\iota}, s_{-\iota}\right) \quad \text { (never-best-rep) }
$$

for each $s_{-\iota} \in S_{-\iota}$.

Remark 1. A Lyapunov-like function is a monotonic function that asymptotically converges to an equilibrium point. The best reply dynamics represent the natural behavior of a Lyapunov-like cost function given by Definition 4. Then, throughout this paper only games with single-value best reply functions will be considered.

For each player $\iota \in \mathcal{N}$ and each strategy-profile of the other players $s_{-\iota} \in S_{-\iota}$, the individual best reply $b_{\iota}$ : $S_{-\iota} \rightarrow 2^{S_{\iota}}$ is assumed to be single valued, i.e.,

$$
b_{\iota}\left(s_{-\iota}\right)=\arg \min _{s_{\iota} \in S_{\iota}} \mathbb{V}\left(s_{\iota}, s_{-\iota}\right) \text {. }
$$

Here $b_{\iota}: S_{-\iota} \rightarrow 2^{S_{\iota}}$ denotes the individual best reply that minimizes the preference ordering over $S_{\iota} \times S_{-\iota}$ of player $\iota$. The best-reply function for a game $G$ is given by $b: S \rightarrow S$ with $b(s)=\prod_{\iota=1}^{n} b_{\iota}\left(s_{-\iota}\right)$. Below we will show that the best reply provides a natural way of thinking about equilibrium points.

Definition 7. A strategy $s_{\iota} \in S_{\iota}$ is said to be strictly dominated if for every strategy-profile of the other players $s_{-\iota}=\left(s_{1}, \ldots, s_{\iota-1}, s_{\iota+1}, \ldots, s_{n}\right)$ there exists a strategy $\delta_{\iota}$ such that $\mathbb{V}\left(\delta_{\iota}, s_{-\iota}\right)<\mathbb{V}\left(s_{\iota}, s_{-\iota}\right)$. We say that, if $s_{\iota}$ is dominated by some $\delta_{\iota}$, then it is dominated, otherwise, then it is undominated.

Theorem 1. Let $G(\mathcal{V}, \mathcal{E})$ be a game graph. If a strategy $s_{\iota}$ is strictly dominated in $S_{\iota}$, it is never a best reply.

Proof. Following the definition of never best reply, given that $s_{\iota}$ is strictly dominated by some $\delta_{\iota}$, we have that $\mathbb{V}_{\iota}\left(\delta_{\iota}, s_{-\iota}\right)<\mathbb{V}_{\iota}\left(s_{\iota}, s_{-\iota}\right)$, which explains the desired statement.

Dominance is not only a sufficient condition for there never being a best reply, but it is also a necessary one.
Corollary 2. Let $G(\mathcal{V}, \mathcal{E})$ be a game graph. If a strategy $\delta_{\iota}$ is dominant in $S_{\iota}$, then it is a best reply.

Definition 8. The dominance solution of game $G(\mathcal{V}, \mathcal{E})$ is defined as the set

$$
D(G)=\bigcap_{l=1}^{\infty} \theta^{l}(S)
$$

such that for $l=0$ we have that $\theta^{0}=S$, and for $l \geq 1$ and for every player $\iota \in \mathcal{N}$ we have that $s_{\iota} \in \theta_{\iota}^{l}$ if there is no $s_{\iota}^{\prime} \in \theta_{\iota}^{l-1}$ such that for all $s_{-\iota} \in \theta_{\iota}^{l-1}$

$$
\mathbb{V}_{\iota}\left(s_{\iota}^{\prime}, s_{-\iota}\right)<\mathbb{V}_{\iota}\left(s_{\iota}, s_{-\iota}\right)
$$

A game graph $G(\mathcal{V}, \mathcal{E})$ is called strictly dominated (dominance solvable) if, and only if, there exists a unique strategy $s \in D(G)$. This means that a game $G(\mathcal{V}, \mathcal{E})$ is a strictly-dominated game if given a sequence of games $G_{0}, \ldots, G_{l}$ satisfies recursively the following conditions:

1. $G_{0}=G$.

2. For a given $j, 0 \leq j \leq l, G_{j+1}$ is a subgame of $G_{j}$ achieved by the elimination of a strictly-dominated strategy from the strategy space of one player in $G_{j}$.

3. The strategy space of each player in $G_{l}$ is of size 1 .

Thus, a game (with the rules discussed above) is obtained by iterated elimination of never best replies from a game $G(\mathcal{V}, \mathcal{E})$ after a number of elimination steps. Note that, in any analysed game $G$, no player $\iota \in \mathcal{N}$ has never best replies and the strategy space of each player in $D(G)$ is of size 1 .

\subsection{Lyapunov game definition and equilibrium uniqueness.}

Definition 9. A Lyapunov game is a game based on the Lyapunov-like function $\mathbb{V}$.

In this case it is possible to specify Definition 9 by establishing that a Lyapunov game is a strictly dominated game in which the iterated elimination of strictly dominated strategies, based on the Lyapunov-like function $\mathbb{V}$, results in a single strategy-profile.

Remark 2. The process of iterated elimination of strategies based on the evolution of the Lyapunov-like function $\mathbb{V}$ can be understood as a formal description of the internal process of the reasoning of a player in which natural common knowledge of the players is that only best replies are chosen

From Definition 4 of the Lyapunov-like function, the following claims hold. 
Remark 3. Let $G(\mathcal{V}, \mathcal{E})$ be a strictly dominated game. The strategy $s^{*} \in S$ is a Lyapunov equilibrium point if and only if $s^{*}$ is a fixed point of the best reply function $b$, i.e., $s^{*} \in b(s)$.

Remark 4. Let $G(\mathcal{V}, \mathcal{E})$ be a Lyapunov game. If the best reply function $b$ associated with $G$ is single valued, then $G$ has a Lyapunov equilibrium point.

Remark 5. Starting from $s_{0}$ and proceeding with the iteration, eventually the trajectory given by

$$
s_{0}<\mathbb{V}_{\iota} s_{1}<\mathbb{V}_{\iota} \cdots<\mathbb{V}_{\iota} s_{m}<\mathbb{V}_{\iota} \cdots
$$

(with $s_{0}, s_{1}, \ldots, s_{m}$ being elements of the trajectory) converges to $s^{*}$, i.e., the optimum trajectory is obtained iteratively. Since at an optimum trajectory an optimum strategy $s^{*}$ holds, we have that

$$
\forall s, s^{*}: \mathbb{V}_{\iota}\left(s^{*}\right)<\mathbb{V}_{\iota}(s) .
$$

Thus, the existence of $s^{*}$ is guaranteed by the Lyapunovlike function where the infimum is asymptotically approached or the minimum is attained.

Lemma 1. Let $G(\mathcal{V}, \mathcal{E})$ be a Lyapunov game. Then, the Lyapunov-like function $\mathbb{V}$ has an asymptotically approached infimum or reaches a minimum.

Proof. Suppose that $s^{*}$ is an equilibrium point. We want to show that $\mathbb{V}$ has an asymptotically approached infimum (or reaches a minimum). Therefore, $s^{*}$ is a sink. Then it follows that the strategy attached to the action(s), following $s^{*}$, is zero. Therefore, let us assume the value of $\mathbb{V}$ cannot be modified. Since the Lyapunov-like function is a decreasing function of the strategies $s \in S$ (by Definition (4), an infimum or a minimum is attained in $s^{*}$.

Theorem 2. A Lyapunov game $G(\mathcal{V}, \mathcal{E})$ has a unique Lyapunov equilibrium point.

Proof. By the definition of a strictly-dominated game $G(\mathcal{V}, \mathcal{E})$, a subgame is achieved by the elimination of a strictly dominated strategy from the strategy space of one player leading to a single strategy profile. Let $s^{*}=$ $\left(s_{\iota}^{*}, s_{-\iota}\right)$ be the profile of strategies that results form the elimination process. Suppose that $s^{* *}=\left(s_{\iota}, s_{-\iota}\right) \neq s^{*}$ is also a Lyapunov equilibrium point. Proceeding with the iteration of the elimination process, the strategy $s_{\iota}$ will be removed (at some time) from the strategy space. Since, at the optimum trajectory the optimum strategy $s^{* *}$ holds, it follows that the Lyapunov-like function satisfies the condition

$$
\mathbb{V}\left(s_{\iota}^{*}, s_{-\iota}\right)<\mathbb{V}\left(s_{\iota}^{\prime}, s_{-\iota}\right)<\mathbb{V}\left(s_{\iota}, s_{-\iota}\right)
$$

for every $s_{-\iota} \in S_{-\iota}$. This is in contradiction with the fact that a Lyapunov-like function is a strictly decreasing function except at the equilibrium point, by Definition 4
Remark 6. Let $G(\mathcal{V}, \mathcal{E})$ be Lyapunov game. Given an arbitrary strategy profile of the players, the best reply dynamics satisfy recursively the following conditions:

1. Choose a player whose strategy is not the best reply to the current strategies of the other players.

2. Change the strategy of that player to the best reply strategy (there is only one).

3. Repeat Steps 1 and 2 until a Lyapunov equilibrium point is reached.

Remark 7. It is important to note that the best reply dynamics represent the monotonic decreasing natural evolution of a Lyapunov-like function.

The best reply is an implementation of a Lyapunovlike function by Definition 6 and then the following property holds

Remark 8. Let $G(\mathcal{V}, \mathcal{E})$ be a Lyapunov game. The best reply dynamics converge to a Lyapunov equilibrium point.

\subsection{Examples.}

Example 1. The Battle of the Sexes is a two-player game used in game theory to conceptualized coordination. The game can be illustrated by a couple. The husband would like to go to a party while the wife would like to go to the opera. Both players prefer engaging in the same activity over going alone. The game is depicted by Table 1 .

Table 1. Game of Example 1.

\begin{tabular}{|c|c|c|}
\hline Wife $\backslash$ Husband & Opera & Party \\
\hline Opera & $2, \mathbf{1}$ & $0, \mathbf{0}$ \\
\hline Party & $0, \mathbf{0}$ & $1, \mathbf{2}$ \\
\hline
\end{tabular}

The game has two pure Lyapunov equilibrium points $(2, \mathbf{1})$ and $(1,2)$. Convergence to one of these Lyapunov equilibrium point by the best reply dynamics is always guaranteed from any initial strategy profile.

Example 2. The repeated Prisoner's Dilemma(PD) (Axelrod, 1984) game with transitions (in the classical formulation this problem is considered as a static one-step game which has nothing in common with transitions) is used here as a first conceptual approximation of an interacting conflict arising between mutual support and selfish exploitation. The same mathematical description finds application in other repeated games dealing with The arms race model and The security model.

The $P D$ game is usually illustrated by a practical situation where two men are arrested for a crime. The police tell each suspect separately that if he testifies against the other, he will be rewarded for defecting him. Each prisoner has two possible strategies: to cooperate (not to 
Table 2. Game of Example 2.

\begin{tabular}{|c|c|c|}
\hline Player $1 \backslash$ Player2 & Cooperate & Defect \\
\hline Cooperate $($ not testify $)$ & $R, \mathbf{R}$ & $S, \mathbf{T}$ \\
\hline Defect $($ testify $)$ & $T, \mathbf{S}$ & $P, \mathbf{P}$ \\
\hline
\end{tabular}

testify) or to defect from the other (testify). If both players defect, there is a mutual punishment (payoff of $P$, the punishment corresponding to mutual defection). If neither testifies, there is a mutual reduction of punishment, resulting in a payoff value of $R$. However, if one testifies and the other does not, the testifier receives considerable punishment reduction (payoff of $S$, the "sucker" payoff for attempting to cooperate against defection), and the other player receives the regular punishment (payoff of $T$, the temptation for defection).

This game has usually two equilibrium points: one non-cooperative (both prisoners testifying) and the other one cooperative (none of the prisoners testifying to the police). Each player wants to minimize the time spent in jail, or, equivalently, maximize the time spent out of jail. Let us suppose that $P, R, S, T$ denote values for time spent out of jail for the next 10 years, such that $T>R>$ $P>S$, where $T=10, R=5, P=3, S=1$.

Each player wants to minimize the time spent in jail. Let us suppose that $T>R>P>S$ and consider the min function (Clempner, 2006) to be a specific (best reply) Lyapunov-like function able to lead a player to an equilibrium point. It is easy to see that we have the structure of a dilemma like the one in the story. On the one hand, suppose that Player 2 does not testify. Then Player 1 obtains $R$ for cooperating and $T$ for defecting, and so he/she is better off defecting, since $T>R$. On the other hand, suppose that Player 2 does testify. Then Player $1 \mathrm{ob}-$ tains $S$ for cooperating and $P$ for defecting, and so he/she is again better off defecting, since $P>S$. The strategy testify (defect) for Player 1 is said to strictly dominate the strategy not testify (cooperate): whatever his/her opponent does, he is better off choosing to testify, rather than to not testify. By symmetry testifying also strictly dominates not testifying for Player 2. Thus two "rational" players will defect and receive a payoff of $(P, P)$.

It is important to note that the unstable strategy cooperate-cooperate with a score of $(R, R)$ is better for both players than the strategy defect-defect with the payoff $(P, P)$. The instability of the cooperate-cooperate strategy means that it is the interest of both players to unilaterally change strategy from cooperate to defect. But if both payers change strategies simultaneously, then they lose, since $R>P$ and $T>P$.

\section{Existence of a Lyapunov-like function}

\subsection{Existence.}

Theorem 3. Let $G(\mathcal{V}, \mathcal{E})$ be a game graph. Then a Lyapunov-like function can be constructed iff $s^{*} \in \mathcal{N}$ is reachable from $s_{0}$.

Proof. $(\Longrightarrow)$ If there exist a Lyapunov-like function $\mathbb{V}$, then, by Definition $4 s^{*}$ is reachable. $(\Longleftarrow)$ By induction, construct the optimal inverse path from $s^{*}$ to $s_{0}$. The node of a system $s_{m}$ is observed in descending order and an edge leading to the strategy profile $s_{m-1}$ is chosen. We choose the trajectory function $\mathbb{V}(s)$ as the best choice set of nodes. We continue this process until $s_{0}$ is reached. Then the trajectory function $\mathbb{V}$ is a Lyapunov-like function.

Remark 9. The goal of the previous theorem is to associate to any game graph $G$ a Lyapunov-like function which monotonically decreases on the trajectories of $G$.

4.2. Lyapunov function construction for repeated (or ergodic) games. Consider a sequence $\left\{s_{m}\right\}_{m \geq 1}$ of strategies $s_{m} \in S$, where $m=1,2, \ldots$ is time (or iterating) index. For these games we will construct a Lyapunovlike function $\mathbb{V}(s)$ (which is, obviously, not unique) as a complex vector-function,

$$
\mathbb{V}(s):=\left(\mathbb{V}_{1}(s), \ldots, \mathbb{V}_{m}(s)\right)^{\top}
$$

where each component $\mathbb{V}_{i}(s)$ is a function of the corresponding cost-value $\mathbb{U}_{i}(s)$, namely,

$$
\mathbb{V}_{i}(s):=\stackrel{\circ}{\mathbb{V}}_{i}\left(\mathbb{U}_{i}(s)\right), \quad i=1, \ldots, m
$$

Here we will show how to construct the functions $\mathbb{V}_{i}\left(\mathbb{U}_{i}\right)$ such that the function $\mathbb{V}(s)$ with the components (13) would satisfy. Conditions 1-4 in Definition 4

Theorem 4. (On a Lyapunov function construction) For repeated (ergodic) games with bounded non-negative cost functions

$$
\begin{aligned}
\mathbb{U}_{i} \in\left[0, \mathbb{U}_{i}^{+}\right], \quad \min _{j \neq i}\left|\mathbb{U}_{i}-\mathbb{U}_{j}\right|= & \varepsilon_{i}>0, \\
& i=1, \ldots, m .
\end{aligned}
$$

within the class of differentiable vector-function $\mathbb{V}_{i}\left(\mathbb{U}_{i}\right)$ whose derivatives satisfy the Lipschitz condition

$$
\left|\frac{\mathrm{d}}{\mathrm{d} \mathbb{U}_{i}} \stackrel{\circ}{V}_{i}\left(\mathbb{U}_{i}^{\prime}\right)-\frac{\mathrm{d}}{\mathrm{d} \mathbb{U}_{i}} \stackrel{\circ}{V}_{i}\left(\mathbb{U}_{i}^{\prime \prime}\right)\right| \leq L\left|\mathbb{U}_{i}^{\prime}-\mathbb{U}_{i}^{\prime \prime}\right|
$$

for all admissible $\mathbb{U}_{i}^{\prime}, \mathbb{U}_{i}^{\prime \prime}, i=1, \ldots, m$, one of the possible Lyapunov-like vector function $\mathbb{V}(s)$ has the compo- 
nents

$$
\begin{aligned}
& \stackrel{\circ}{\mathbb{V}}_{i}\left(\mathbb{U}_{i}\right)=\left\{\begin{array}{cc}
\stackrel{\circ}{\mathbb{V}}_{i}\left(\mathbb{U}_{i}\left(s_{m-1}\right)\right) \exp \left\{-\left(\alpha_{i} / \gamma_{m, i}\right)\right. \\
\left.\mathbb{U}_{i}\left(s_{m-1}\right)\right\} & \text { if } \gamma_{i}>\varepsilon_{i}>0, \\
-\stackrel{\circ}{\beta}_{i} / \alpha_{i} \stackrel{\circ}{\mathbb{V}}_{i}\left(\mathbb{U}_{i}\left(s_{m-1}\right)\right) & \text { if } \gamma_{i} \leq 0,
\end{array}\right. \\
& \begin{aligned}
\gamma_{m, i}: & :=\Delta \mathbb{U}_{i}\left(s_{m}, s_{m-1}\right)=\mathbb{U}_{i}\left(s_{m}\right)-\mathbb{U}_{i}\left(s_{m-1}\right), \\
\dot{\beta}_{i} & :=\frac{L}{2}\left(\mathbb{U}_{i}^{+}\right)^{2},
\end{aligned}
\end{aligned}
$$

with the initial condition $\mathbb{V}_{i}\left(s_{0}\right)$ satisfying

$$
\begin{aligned}
\stackrel{\circ}{\mathbb{V}}_{i}\left(s_{0}\right) \geq & \frac{1}{2 \alpha_{i}} L\left(\mathbb{U}_{i}^{+}\right)^{2} \exp \left(\frac{\alpha_{i}}{\varepsilon_{i}}\right) \\
& -\frac{\stackrel{\rho}{i}_{i}}{\alpha_{i}}>0
\end{aligned}
$$

and any $\alpha_{i}$ such that

$$
1 \geq \frac{\alpha_{i}}{2 \varepsilon_{i}^{2}} \exp \left(\left(\frac{\alpha_{i}}{\varepsilon_{i}}\right)\left(1+\mathbb{U}_{i}^{+}\right)\right)\left(\mathbb{U}_{i}^{+}\right)^{2}
$$

when $\left|\Delta \mathbb{U}_{i}\left(s_{m}, s_{m-1}\right)\right| \geq \varepsilon_{i}>0, i=1, \ldots, m$ for any $\gamma_{i} \neq 0$, which implies

$$
\begin{aligned}
\mathbb{V}_{i}\left(s_{m}\right):= & \stackrel{\mathbb{V}}{i}_{i}\left(\mathbb{U}_{i}\left(s_{m}\right)\right) \\
\leq & \left(1-\alpha_{i}\right) \mathbb{V}_{i}\left(s_{m-1}\right) \chi\left(\left|\gamma_{m, i}\right| \geq \varepsilon_{i}>0\right) \\
& +\mathbb{V}_{i}\left(s_{m-1}\right)\left[1-\chi\left(\left|\gamma_{m, i}\right| \geq \varepsilon_{i}>0\right)\right] \\
= & \mathbb{V}_{i}\left(s_{m-1}\right)\left[1-\alpha_{i} \chi\left(\left|\gamma_{m, i}\right| \geq \varepsilon_{i}>0\right)\right] \\
\leq & \mathbb{V}_{i}\left(s_{m-1}\right) \alpha_{i} \in(0,1) .
\end{aligned}
$$

Therefore,

$$
\tilde{\mathbb{V}}_{i}\left(\mathbb{U}_{i}\right) \leq \stackrel{\circ}{V}_{i}\left(\mathbb{U}_{i}\left(s_{m-1}\right)\right) \exp \left(-\frac{\alpha_{i}}{\gamma} \underset{m, i}{\mathbb{U}_{i}}\right)
$$

and hence we may take

$$
\stackrel{\circ}{V}_{i}\left(\mathbb{U}_{i}\right)=\stackrel{\circ}{V}_{i}\left(\mathbb{U}_{i}\left(s_{m-1}\right)\right) \exp \left(-\frac{\alpha_{i}}{\gamma} \underset{m, i}{\mathbb{U}_{i}}\right)-\frac{\stackrel{\circ}{\beta}_{i}}{\alpha_{i}}
$$

Since $\left|\gamma_{i}\right| \leq \mathbb{U}_{i}^{+}$, in order to guarantee that $\stackrel{\circ}{V}_{i}\left(\mathbb{U}_{i}\right) \geq$ 0 , the value $\tilde{\mathbb{V}}_{i}(0)$ should satisfy

$$
\begin{aligned}
\tilde{\mathbb{V}}_{i}\left(s_{0}\right) \exp \left(-\frac{\alpha_{i}}{\gamma_{m, i}} \mathbb{U}_{i}\right)-\frac{\stackrel{\circ}{i}_{i}}{\alpha_{i}} \\
\quad \geq \tilde{\mathbb{V}}_{i}\left(s_{0}\right) \exp \left(-\frac{\alpha_{i}}{\varepsilon_{i}}\right)-\frac{1}{2 \alpha_{i}} L\left(\mathbb{U}_{i}^{+}\right)^{2} \geq 0
\end{aligned}
$$

or, equivalently,

$$
\tilde{\mathbb{V}}_{i}\left(s_{0}\right) \geq \frac{1}{2 \alpha_{i}} L\left(\mathbb{U}_{i}^{+}\right)^{2} \exp \left(\frac{\alpha_{i}}{\varepsilon_{i}}\right) .
$$

Taking into account that the function $\exp \left\{-\left(\alpha_{i} / \gamma_{m, i}\right) \mathbb{U}_{i}\right\} \quad$ is twice differentiable, we may conclude that

$$
\begin{aligned}
L & =\max _{\mathbb{U}_{i}}\left|\frac{d^{2}}{d \mathbb{U}_{i}^{2}} \stackrel{\circ}{i}_{i}\left(\mathbb{U}_{i}\right)\right| \\
& =\max _{\mathbb{U}_{i}}\left[\left(\frac{\alpha_{i}}{\gamma_{m, i}}\right)^{2} \tilde{\mathbb{V}}_{i}\left(s_{0}\right) \exp \left(-\frac{\alpha_{i}}{\gamma_{m, i}} \mathbb{U}_{i}\right)\right] \\
& =\left(\frac{\alpha_{i}}{\varepsilon_{i}}\right)^{2} \tilde{\mathbb{V}}_{i}\left(s_{0}\right) \exp \left(\frac{\alpha_{i}}{\varepsilon_{i}} \mathbb{U}_{i}^{+}\right)
\end{aligned}
$$

Proof. By the condition (15) and in view of Lemma 21.1 on a finite increment by Poznyak (2008), it follows that

$$
\begin{aligned}
\stackrel{\circ}{V}_{i}\left(\mathbb{U}_{i}\left(s_{m}\right)\right)= & \stackrel{\circ}{\mathbb{V}}_{i}\left(\mathbb{U}_{i}\left(s_{m-1}\right)\right. \\
& \left.+\Delta \mathbb{U}_{i}\left(s_{m}, s_{m-1}\right)\right) \leq \stackrel{\circ}{V}_{i}\left(\mathbb{U}_{i}\left(s_{m-1}\right)\right) \\
& +\frac{\mathrm{d}}{\mathrm{d} \mathbb{U}_{i}} \stackrel{\circ}{V}_{i}\left(\mathbb{U}_{i}\left(s_{m-1}\right)\right) \Delta \mathbb{U}_{i}\left(s_{m}, s_{m-1}\right) \\
& +\frac{L}{2}\left\|\Delta \mathbb{U}_{i}\left(s_{m}, s_{m-1}\right)\right\|^{2} .
\end{aligned}
$$

For any fixed number $m$, write

$$
\gamma_{m, i}:=\Delta \mathbb{U}_{i}\left(s_{m}, s_{m-1}\right),
$$

and let us try to find a function $\mathbb{V}_{i}\left(\mathbb{U}_{i}\right)$ which satisfies

$$
\frac{\mathrm{d}}{\mathrm{d} \mathbb{U}_{i}} \stackrel{\circ}{V}_{i}\left(\mathbb{U}_{i}\right) \gamma_{m, i}+\frac{L}{2} \gamma_{m, i}^{2} \leq-\alpha_{i} \stackrel{\circ}{V}_{i}\left(\mathbb{U}_{i}\right)
$$

(a) Suppose now that $\gamma_{m, i} \geq \varepsilon_{i}>0$. Then the function $\tilde{\mathbb{V}}_{i}\left(\mathbb{U}_{i}\right):=\stackrel{\circ}{\mathbb{V}}_{i}\left(\mathbb{U}_{i}\right)+\stackrel{\circ}{\beta}_{i} / \alpha_{i}$ satisfies

$$
\frac{\mathrm{d}}{\mathrm{d} \mathbb{U}_{i}} \tilde{\mathbb{V}}_{i}\left(\mathbb{U}_{i}\right) \gamma_{m, i} \leq-\alpha_{i} \tilde{\mathbb{V}}_{i}\left(\mathbb{U}_{i}\right) .
$$

which implies (18) guaranteeing the nonnegativity of $\stackrel{\circ}{V}_{i}\left(\mathbb{U}_{i}\right)$. Therefore, substituting (20) into (21) leads to

$$
\stackrel{\circ}{V}_{i}\left(\mathbb{U}_{i}\left(s_{m}\right)\right) \leq\left(1-\alpha_{i}\right) \mathbb{V}_{i}\left(s_{m-1}\right) \chi\left(\gamma_{m, i} \geq \varepsilon_{i}>0\right) .
$$

(b) Consider the case $\gamma_{m, i} \leq 0$, which, together with (20), implies that

$$
\stackrel{\mathbb{V}}{i}_{i}\left(\mathbb{U}_{i}\left(s_{m}\right)\right)=\stackrel{\mathscr{V}}{i}_{i}\left(\mathbb{U}_{i}\left(s_{m-1}\right)\right) \chi\left(\gamma_{m, i} \leq 0\right)
$$

(c) The case $0 \leq \gamma_{m, i}<\varepsilon_{i}$ is excluded by the assumption (14).

Combining the recursions in (a) and (b), we obtain (14). The theorem is thus proven.

Remark 10. By the inequality $1-x \leq \exp (-x)$, it follows that

$$
\begin{aligned}
\mathbb{V}_{i}\left(s_{m}\right) & =\mathbb{V}_{i}\left(s_{m-1}\right)\left[1-\alpha_{i} \chi\left(\gamma_{m, i} \geq \varepsilon_{i}>0\right)\right] \\
& \leq \mathbb{V}_{i}\left(s_{m-1}\right) \exp \left(-\alpha_{i} \chi\left(\gamma_{m, i} \geq \varepsilon_{i}>0\right)\right) \\
& \leq \cdots \\
& \leq \mathbb{V}_{i}\left(s_{0}\right) \\
& \exp \left(-\alpha_{i} \sum_{t=1}^{m}\left(\chi\left(\gamma_{t, i} \geq \varepsilon_{i}>0\right)\right)\right) \rightarrow 0
\end{aligned}
$$


as $m \rightarrow \infty$ if

$$
\sum_{t=1}^{\infty} \chi\left(\gamma_{t, i} \geq \varepsilon_{i}>0\right)=\infty
$$

Remark 11. Based on the above remark, the Lyapunov function can be suggested as

$$
\begin{aligned}
& \mathbb{V}_{i}\left(s_{m}\right) \\
& =\mathbb{V}_{i}\left(s_{0}\right) \exp \left(-\alpha_{i} \sum_{t=1}^{m}\left(\chi\left(\gamma_{t, i} \geq \varepsilon_{i}>0\right)\right)\right) .
\end{aligned}
$$

Example 3. Consider the repeated Prisoner's Dilemma game with the following cost functions:

$$
S:=\{C C, C D, D C, D D\},
$$

Table 3. Game of Example 3.

\begin{tabular}{|c|c|c|}
\hline Player $1 \backslash$ Player2 & Cooperate & Defect \\
\hline Cooperate $($ not testify $)$ & $R, \mathbf{R}$ & $S, \mathbf{T}$ \\
\hline Defect $($ testify $)$ & $T, \mathbf{S}$ & $P, \mathbf{P}$ \\
\hline
\end{tabular}

$$
\begin{array}{rlrl}
\mathbb{U}_{1}(C C) & =5, & \mathbb{U}_{1}(C D)=1, \\
\mathbb{U}_{1}(D C)=10, & \mathbb{U}_{1}(D D)=3, \\
\mathbb{U}_{2}(C C)=5, & \mathbb{U}_{2}(C D)=10, \\
\mathbb{U}_{2}(D C)=1, & \mathbb{U}_{2}(D D)=3 .
\end{array}
$$

In this case,

$$
\varepsilon_{1}=\varepsilon_{2}=2, \quad \mathbb{U}_{1}^{+}=\mathbb{U}_{2}^{+}=10, \quad \alpha_{1}=\alpha_{2}=\frac{1}{15} .
$$

For a given initial condition, take $\tilde{\mathbb{V}}_{1}\left(s_{0}\right)=100$ and $\tilde{\mathbb{V}}_{2}\left(s_{0}\right)=100$. The following results have been obtained:

- In Figs. 1 and 3 the state-value function behavior is shown (where during game repetition the states of the players fluctuate according to the given strategies) showing a completely non monotonic behavior.

- In Figs. 2 and 4 the corresponding Lyapunovlike functions are plotted definitely demonstrating a monotonic decreasing behavior.

Example 4. Consider the Battle of the Sexes game with the following cost functions:

$$
\begin{array}{rlrl}
S: & =\{O O, O P, P O, P P\}, \\
\mathbb{U}_{1}(O O) & =2, & \mathbb{U}_{1}(O P)=0, \\
\mathbb{U}_{1}(P O) & =0, & \mathbb{U}_{1}(P P)=1, \\
\mathbb{U}_{2}(O O) & =1, & \mathbb{U}_{2}(O P)=0, \\
\mathbb{U}_{2}(P O) & =0, & \mathbb{U}_{2}(P P)=2 .
\end{array}
$$

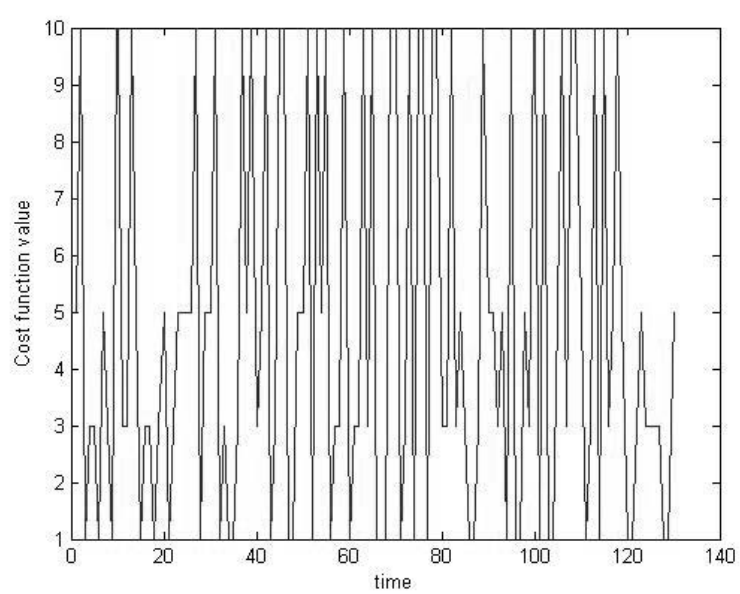

Fig. 1. Cost function for Prisoner's Dilemma Player 1

In this case,

$$
\varepsilon_{1}=\varepsilon_{2}=1, \quad \mathbb{U}_{1}^{+}=\mathbb{U}_{2}^{+}=2, \quad \alpha_{1}=\alpha_{2}=\frac{1}{4} .
$$

For a given initial condition, take $\tilde{\mathbb{V}}_{1}\left(s_{0}\right)=1000$ and $\tilde{\mathbb{V}}_{2}\left(s_{0}\right)=1000$. The following results have been obtained:

- In Figs. 5 and 7 the state-value function behavior is shown (where during game repetition the states of the players fluctuate according to the given strategies) showing a completely nonmonotonic behavior.

- In Fig. 6 and 8 the corresponding Lyapunov-like functions are plotted definitely demonstrating a monotonic decreasing behavior.

\subsection{Completeness.}

Proposition 2. (Cauchy criterion) Let $G(\mathcal{V}, \mathcal{E})$ be a Lyapunov game and $\mathbb{V}$ its corresponding Lyapunov-like function. The realized trajectory $s_{0}, s_{1}, \ldots, s_{m}$ converges to some value if and only if the following holds: for every $\delta>0$ we can find $M$ such that $\left|s_{l}-s_{m}\right|<\delta$ whenever $m, l>M$.

Proof. Since $\exists \epsilon_{i}:\left|\mathbb{V}\left(s_{i+1}\right)-\mathbb{V}\left(s_{i}\right)\right|>\epsilon_{i}$ (with $\epsilon_{i}>0$ ) and $s^{*} \leq_{\mathbb{V}} s$, there are $m, l>M$ such that $\left|s_{l}-s_{m}\right|<\delta$ and $\delta \rightarrow 0$. It is clear that the Cauchy criterion is satisfied and the realized trajectory has a limit.

Theorem 5. Let $G(\mathcal{V}, \mathcal{E})$ be a Lyapunov game, and $\mathbb{V}$ its corresponding Lyapunov-like function. Let $s_{0}, s_{1}, \ldots, s_{m}$ be a realized trajectory which converges to $s^{*}$ such that

$$
\exists \epsilon_{i}:\left|\mathbb{V}_{\iota}\left(s_{i+1}\right)-\mathbb{V}_{\iota}\left(s_{i}\right)\right|>\epsilon_{i}\left(\text { with }_{i}>0\right) .
$$

Furthermore, set $\epsilon=\min \left\{\epsilon_{i}\right\}$. Then the best reply dynamics converge to a Lyapunov equilibrium point 


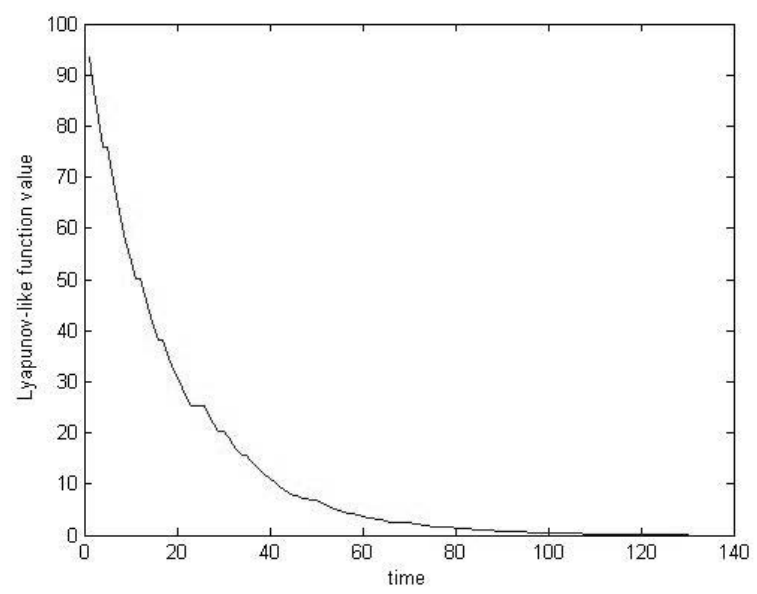

Fig. 2. Lyapunov-like function for Prisoner's Dilemma Player 1.

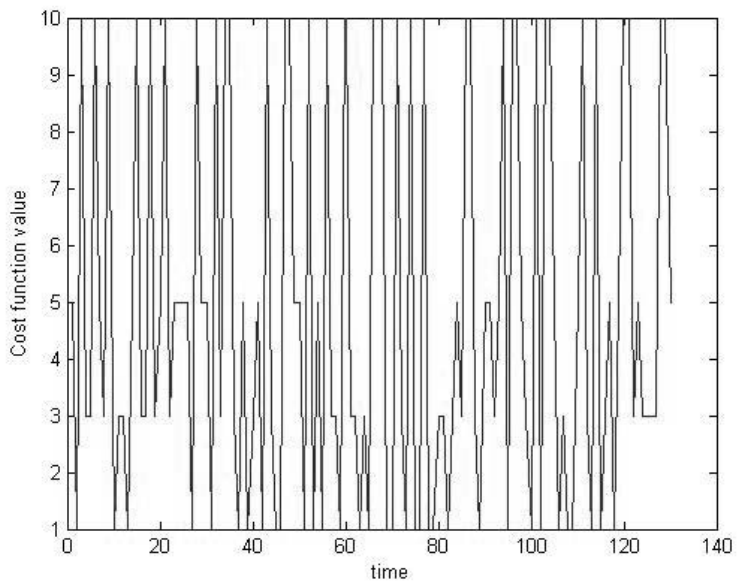

Fig. 3. Cost function for Prisoner's Dilemma Player 2.

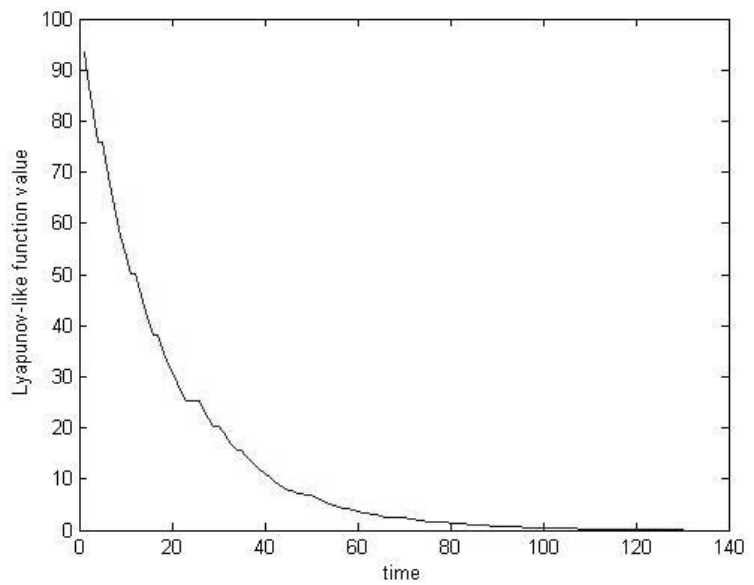

Fig. 4. Lyapunov-like function for Prisoner's Dilemma Player 2.

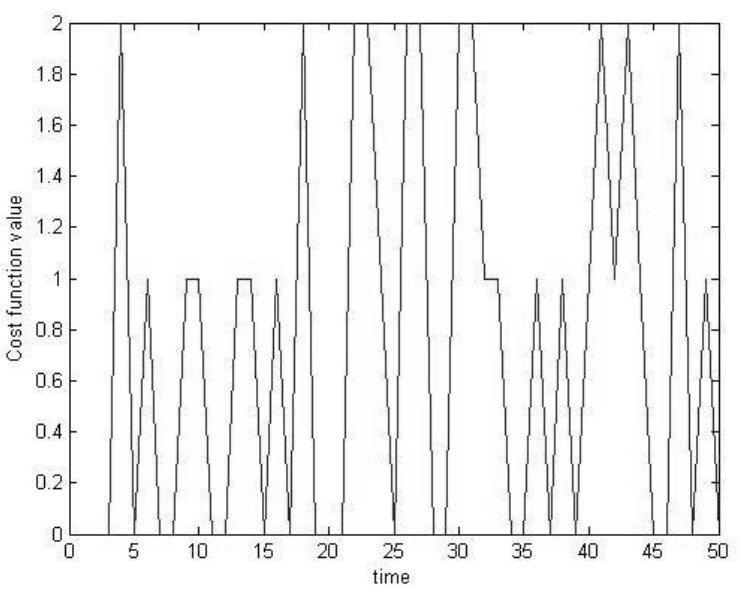

Fig. 5. Cost function for Battle of the Sexes Player 1.

$s^{*}$ within $O\left(\max \left(\mathbb{V}_{\iota}\left(s_{0}\right) / \epsilon\right)\right)$, where $\mathbb{V}_{\iota}\left(s_{0}\right)$ is the initial value of the Lyapunov-like function.

Proof. Suppose that $G(\mathcal{V}, \mathcal{E})$ is not bounded. Thus $s^{*}$ is never reached. Then $s^{*}$ is not the last state in the strictly dominated game $G$. Hence it is possible to find some output transition to $s^{*}$. Since at the optimum trajectory the optimum strategy $s^{*}$ holds, we have that the Lyapunovlike function satisfies $\mathbb{V}(s)<\mathbb{V}\left(s^{*}\right)$. Therefore, it is possible to reduce the trajectory function value over $s^{*}$ by at least $\epsilon$. As a result, it is possible to obtain a lower value than $C$ (that is a contradiction). Then, for a player $\iota$, the equilibrium point $s^{*}$ is reached in a time step bounded by $O\left(\mathbb{V}_{\iota}\left(s_{0}\right) / \epsilon\right)$.

Proposition 3. Let $G(\mathcal{V}, \mathcal{E})$ be a Lyapunov game. Then, $\mathbb{V}$ converges to an equilibrium point $s^{*}$.

Proof. We have to show that $\mathbb{V}_{\iota}$ converges. By the previous theorem the equilibrium point $s^{*}$ is reached in a time step bounded by $O\left(\max \left(\mathbb{V}_{\iota}\left(s_{0}\right) / \epsilon\right)\right)$. Therefore, $\mathbb{V}_{\iota}$ converges to $s^{*}$.

This paper considers games where each player can observe other players' current-period strategies and not all players may revise their strategies in every period. In every period, players decide whether to monitor other players and whom to monitor. The following computational complexity for finding a Lyapunov equilibrium point holds.

Theorem 6. Let $G(\mathcal{V}, \mathcal{E})$ be Lyapunov game. The Lyapunov best reply dynamics converge to a Lyapunov equilibrium point in $O\left(\sum_{\iota=1}^{l}\left(\left|\mathcal{E}_{\iota}\right|\right)\right)$ steps, where $\left|\mathcal{E}_{\iota}\right|$ is the size of the edges for player $\iota$.

Proof. Given a game graph $G(\mathcal{V}, \mathcal{E})$, let $s_{i}$ be a strategy strictly dominated by strategy $s_{j}$. Then the edge $\left(s_{i}, s_{j}\right)$ means that $s_{j}$ has a lower payoff than $s_{i}$, i.e., $\mathbb{V}\left(s_{j}\right)<$ 
$\mathbb{V}\left(s_{i}\right)$ means that the node $s_{j}$ represents the result of minimizing the cost-function. Suppose that Player $\iota$ observes

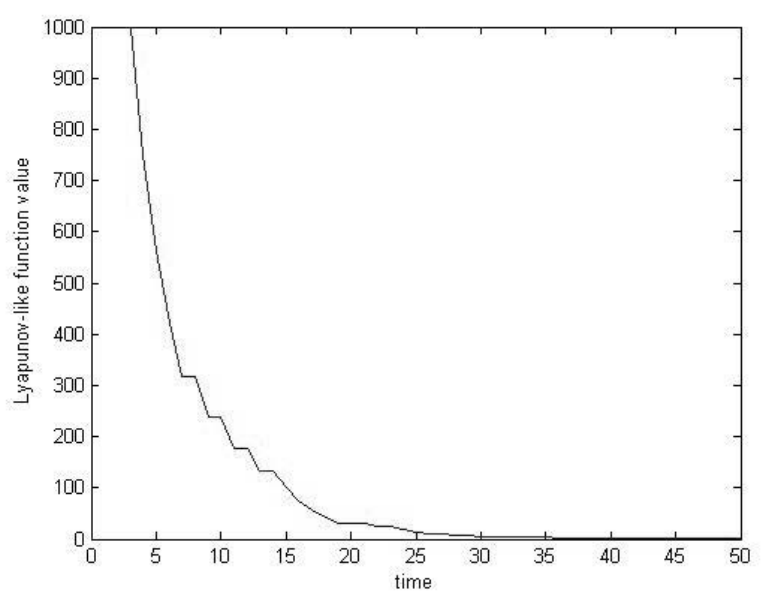

Fig. 6. Lyapunov-like function for Battle of the Sexes Player 1.

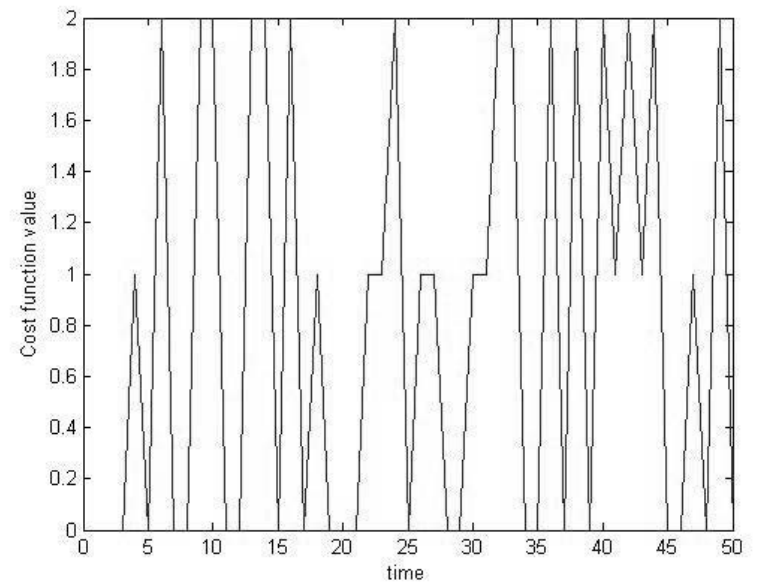

Fig. 7. Cost function for Battle of the Sexes Player 2.



Fig. 8. Lyapunov-like Function for Battle of the Sexes Player 2. other players' current-period strategies and tries to play strategy $s_{i}$. So, Player $\iota$ must choose a different strategy because $s_{i}$ is strictly-dominated by strategy $s_{j}$. By the best reply dynamics in Definition 6, the predecessors of $s_{j}$ in the game graph $G$ will be eliminated and $s_{i}$ and its previous nodes will not be selected again. Continuing with the process, each player can observe other players' current-period strategies but not all players may revise their strategies in every period. After $l$ players, an arbitrary strategy $s$ will not be chosen again because it is strictly dominated. As a result, the strategy selection process for player $\iota$ is restricted to the size of the edges $\left(\left|\mathcal{E}_{\iota}\right|\right)$ in the game graph $G$. Then the Lyapunov best reply dynamics converge to a Lyapunov equilibrium point in $O\left(\Sigma_{\iota=1}^{l}\left(\left|\mathcal{E}_{\iota}\right|\right)\right)$ steps.

\section{Conclusion and future work}

We introduced the concept of Lyapunov game as a subclass of strictly dominated games and potential games:

- In Lyapunov games, natural existence of the equilibrium point is assured by definition, in contrast to the fact that a Nash equilibrium must satisfy Kakutani's fixed-point theorem.

- Convergence is also guaranteed to exist. A Lyapunov-like function definitely converges to a Lyapunov equilibrium point.

- A Lyapunov equilibrium point presents properties of stability that are not necessarily present at a Nash equilibrium point.

- In this sense, a Lyapunov equilibrium point is a Nash equilibrium point, but the opposite is not necessarily true.

We present here an approach to finding an equilibrium point using a Lyapunov-like function as the best reply dynamics in strictly dominated games. It begins with a given strategy profile of the players, in each step letting some player to choose a strategy using a Lyapunov-like function that represents the best reply to the current strategies of the others.

By definition, a Lyapunov-like function monotonically decreases and converges to a Lyapunov equilibrium point identified by the sink of the game graph. It is important to note that in previous work this convergence has not been guaranteed even if the Nash equilibrium point exists. The best reply dynamics result in a natural implementation of the behavior of a Lyapunov-like function. Therefore, a Lyapunov game has also the benefit that it is common knowledge of the players that only best replies are chosen. By the natural evolution of a Lyapunov-like function, a strategy played once is not played again. 
Since a Lyapunov-like function is to respect the system constraints, it will lead the system from the source state to the equilibrium point. Each optimal action applied produces a monotonic progress towards an equilibrium point. As a result, the process converges and will certainly happen in a forward sense.

We also show that the best reply dynamics using a Lyapunov-like function guarantee that convergence in simultaneous order is $O\left(\max \left(\mathbb{V}_{\iota}\left(s_{0}\right) / \epsilon\right)\right)$, where $\mathbb{V}_{\iota}\left(s_{0}\right)$ is the initial value of the Lyapunov-like function and in asynchronous order is $O\left(\sum_{\iota=1}^{l}\left(\left|\mathcal{E}_{\iota}\right|\right)\right)$ steps where $\left|\mathcal{E}_{\iota}\right|$ is the size of the edges for Player $\iota$ in the game graph. This computational complexity complements the result of the completeness of computing a Nash equilibria obtained by Goemans et al. (2005), Fabrikant et al. (2004) as well as Fabrikant and Papadimitriou (2008) for potential games.

There are a number of questions related to classical game theory that may in the future be addressed satisfactorily within this framework. In this sense, as future work we will extend the present idea to support the shortest path problem (Tarapata, 2007), methods for computing Pareto sets (Toth and Kreinovich, 2009) and its relationship with Lyapunov and dynamic routing protocols (Griffin and Shepherd, 2002).

\section{References}

Axelrod, R. (1984). The Evolution of Cooperation, Basic Books, New York, NY.

Bernheim, B.D. (1984). Rationalizable strategic behavior, Econometrica 52(4): 1007-1028.

Chen, X. and Deng, X. (2006). Setting the complexity of 2-player Nash equilibrium, Proceedings of the 47th Annual IEEE Symposium on Foundations of Computer Science, FOCS 2006, Berkeley, CA, USA, pp. 261-270.

Chen, X., Deng, X. and Tengand, S.-H. (2006). Computing Nash equilibria: Approximation and smoothed complexity, Proceedings of the 47th Annual IEEE Symposium on Foundations of Computer Science, FOCS 2006, Berkeley, CA, USA, pp. 603-612.

Clempner, J. (2006). Modeling shortest path games with Petri nets: A Lyapunov based theory, International Journal of Applied Mathematics and Computer Science 16(3): 387397.

Daskalakis, C., Goldberg, P. and Papadimitriou, C. (2006a). The complexity of computing a Nash equilibrium, Proceedings of the 38th ACM Symposium on Theory of Computing, STOC 2006, Seattle, WA, USA, pp. 71-78.

Daskalakis, C., Mehta, A. and Papadimitriou, C. (2006b). A note on approximate Nash equilibria, Proceedings of the 2nd Workshop on Internet and Network Economics, WINE 06, Patras, Greece, pp. 297-306.

Fabrikant, A. and Papadimitriou, C. (2008). The complexity of game dynamics: BGP oscillations, sink equilibria, and beyond, Proceedings of the 19th Annual ACM-SIAM Sympo- sium on Discrete Algorithms, SODA 2008, San Francisco, CA, USA, pp. 844-853.

Fabrikant, A., Papadimitriou, C. and Talwar, K. (2004). The complexity of pure Nash equilibria, Proceedings of the 36th ACM Symposium on Theory of Computing, STOC 2006, Chicago, IL, USA, pp. 604-612.

Goemans, M., Mirrokni, V. and Vetta, A. (2005). Sink equilibria and convergence, Proceedings of the 46th IEEE Symposium on Foundations of Computer Science, FOCS 2008, Pittsburgh, PA, USA, pp. 142-154.

Griffin, T.G. and Shepherd F.B. and Wilfong, G.W. (2002). The stable paths problem and interdomain routing, IEEE/ACM Transactions on Networking 10(2): 232-243.

Kakutani, S. (1941). A generalization of Brouwer's fixed point theorem, Duke Journal of Mathematics 8(3): 457-459.

Kontogiannis, S., Panagopoulou, P. and Spirakis, P. (2006) Polynomial algorithms for approximating nash equilibria of bimatrix games, Proceedings of the 2nd Workshop on Internet and Network Economics, WINE 06, Patras, Greece, pp. 286-296.

Lakshmikantham, V., Matrosov, V. and Sivasundaram, S. (1991) Vector Lyapunov Functions and Stability Analysis of Nonlinear Systems, Kluwer Academic Publication, Dordrecht.

Lipton, R.J., Markakis, E. and Mehta, A. (2003). Playing large games using simple strategies, Proceedings of the 4th ACM Conference on Electronic Commerce, EC 2003, San Diego, CA, USA, pp. 36-41.

Mirrokni, V. and Vetta, A. (2004). Convergence issues in competitive games, Proceedings of the 7th International Workshop on Approximation Algorithms for Combinatorial Optimization Problems, APPROX 2004, Cambridge, MA, USA, pp. 183-194.

Moulin, H. (1984). Dominance solvability and Cournot stability, Mathematical Social Sciences 7(1): 83-102.

Myerson, R. B. (1978). Refinements of the Nash equilibrium concept, International Journal of Game Theory 7(2): 7380 .

Nash, J. (1951). Non-cooperative games, Annals of Mathematics 54(2): 287-295.

Nash, J. (1996). Essays on Game Theory, Elgar, Cheltenham.

Nash, J. (2002). The Essential John Nash, H.W. Kuhn and S. Nasar, Princeton, NJ.

Pearce, D. (1984). Rationalizable strategic behavior and the problem of perfection, Econometrica 52(4): 1029-1050.

Poznyak, A.S. (2008). Advance Mathematical Tools for Automatic Control Engineers, Vol. 1: Deterministic Techniques, Elsevier, Amsterdam.

Poznyak, A.S., Najim, K. and Gomez-Ramirez, E. (2000). SelfLearning Control of Finite Markov Chains, Marcel Dekker, New York, NY.

Selten, R. (1975). Reexamination of the perfectness concept for equilibrium points in extensive games, International Journal of Game Theory 4(1): 25-55. 
Tarapata, Z. (2007). Selected multicriteria shortest path problems: An analysis of complexity, models and adaptation of standard algorithms, International Journal of Applied Mathematics and Computer Science 17(2): 269-287, DOI: 10.2478/v10006-007-0023-2.

Topkis, D. (1979). Equilibrium points in nonzero-sum n-persons submodular games, SIAM Journal of Control and Optimization 17(6): 773-787.

Toth, B. and Kreinovich, V. (2009). Verified methods for computing Pareto sets: General algorithmic analysis, International Journal of Applied Mathematics and Computer Science 19(3): 369-380, DOI: 10.2478/v10006/009-0031-5.



Julio B. Clempner has ten-year experience in the field of management consulting. Currently, he specializes in the application of high technology and is involved in providing e-business solutions including e-business strategies, management of technology, operations strategies, project management, continuous quality improvement, and managing total quality transformation. Dr. Clempner's research interests are focused on justifying and introducing the Lyapunov equilibrium point in shortest-path decision processes and shortest-path game theory. This interest has led to several streams of research. One is focused on the use of Markov decision processes for formalizing the previous ideas, changing the Bellman equation by a Lyapunov-like function. A second stream is on the use of Petri nets as a language for modeling decision processes and game theory introducing colors, hierarchy, etc. The final stream examines the possibility to meet modal logic, decision processes and game theory. Dr. Julio Clempner is a member of the Mexican National System of Researchers (SNI).

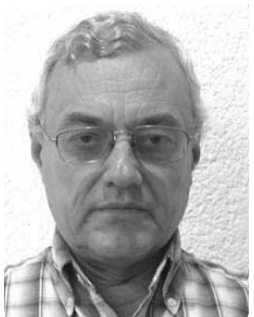

Alexander S. Poznyak graduated from Moscow Physical Technical Institute in 1970. He earned his Ph.D. and D.Sc. degrees from the Institute of Control Sciences of the Russian Academy of Sciences in 1978 and 1989, respectively. From 1973 up to 1993 he served at this institute as a researcher and a leading researcher, and in 1993 he accepted the post of a full professor (3-E) at the Center for Research and Advanced Studies (Cinvestav), National Polytechnic Institute, Mexico. Presently, he is the head of the Automatic Control Department. He has supervised 33 Ph.D. theses (25 in Mexico), and published more than 140 papers in various international journals, as well as nine books. He is a member of the Mexican Academy of Sciences and a member of the Mexican National System of Researchers (SNI-3), an associated editor of the Ibeamerican International Journal on Computations and Systems, and a member of the Evaluation Committee of SNI (Ministry of Science and Technology) responsible for engineering science and technology foundation in Mexico.

Received: 26 January 2010

Revised: 17 August 2010

Re-revised: 15 October 2010 